\title{
Why Bother With a Computerized Scheduling System?
}

\author{
Ronald Arenson
}

\begin{abstract}
Patient scheduling is often included as a module available in modern information management systems. The functionality of a scheduling module can vary greatly. A complete scheduling system combines rules related to each examination room with rules concerning the procedure itself. Duplicate exams can be avoided while picking the most optimal time slot available. The benefits of such a scheduling system include reduction in scheduling delays, a reduction in patient waiting time, improved patient through-put, improved clinical history information, and improved billing accuracy and completeness.

๑) 1988 by W.B. Saunders Company.
\end{abstract}

KEY WORDS: Examination dictionary, film library.

$\mathbf{I}$ NFORMATION management systems for radiology first appeared in the early 1970 s and have become widely used in the last few years. Many commercial systems are now available that offer such functions as registration, scheduling, film library management, patient tracking, reporting, billing, and management statistics. Although many of these commercial systems seem to offer similar functions, there are often significant differences. One area in which these systems differ greatly is in scheduling.

The simplest form of scheduling presents the computerized version of a schedule book into which patients may be logged. This approach is similar to the scheduling of seats on an airplane. At the other extreme is a sophisticated scheduling module first developed at Massachusetts General Hospital (MGH) and then implemented at the Hospital of the University of Pennsylvania (HUP). ${ }^{1-4}$

This complex scheduling system combines a set of examination-specific parameters with a room matrix to select the most appropriate time and date for a particular examination. The exam-specific parameters include the primary and alternate rooms best suited for the procedure, preparation time, earliest and latest times

From the Department of Radiology, Hospital of the University of Pennsylvania, Philadelphia.

Address reprint requests to Ronald Arenson, MD, Professor of Radiology, Department of Radiology, Hospital of the University of Pennsylvania, 3400 Spruce St, Philadelphia, PA 19104.

(c) 1988 by W.B. Saunders Company.

0897-1889/88/0101-0001\$03.00/0 the exams should be performed, and the length of the examination in minutes (Fig 1). These parameters are merged in the scheduling algorithms with the room matrix, which consists of codes for each room for each day of the week. The coded days are divided into five-minute increments. These codes represent categories of patients such as inpatients or outpatients (Fig 2). These codes can also be inclusive or exclusive groupings which may, for example, reserve slots for emergency room patients while allowing them to be scheduled in general outpatient slots if such spaces are open.

The room group for each type of examination in the exam dictionary lists the rooms in which the examination can be performed in the order of their ability to provide the best circumstances for the exam. In addition, ten minutes are added for stretcher patients, while five minutes are subtracted for ambulatory outpatients if their examinations are longer than 15 minutes.

In addition to the aforementioned rules for the scheduling process, there are a set of conflict checks which are also based on indicators in the exam dictionary. Whenever a scheduled examination appears to be similar to an examination performed in the recent past or is already scheduled for the future, a warning is displayed to reduce unnecessary repeat examinations. In addition, there is a sequence number that determines the appropriate order when several examinations are scheduled at the same time. This insures that the intravenous urogram is performed before the barium enema, which would be followed by the upper gastrointestinal exam.

If the radiologist must approve the exam before it is scheduled, a flag in the exam dictionary would so indicate and a message would be presented to the scheduler as a reminder. If the chart is desired for inpatients, an indicator in the exam dictionary would cause the appropriate message to print on the transportation notice.

If an examination is scheduled for the same day, the exam documents would be printed immediately and would include a flash card, examination card, and a transportation notice (Fig 3). The film library pull notice prints the appropriate location to provide the film librarians an opportunity to pull the previous films for 
Fig 1. Exam dictionary example. These data elements for each procedure type are used by programs to schedule exams. This dictionary or tabledriven process allows the user to easily modify entries whenever appropriate. Note the conflict checks and billing data.

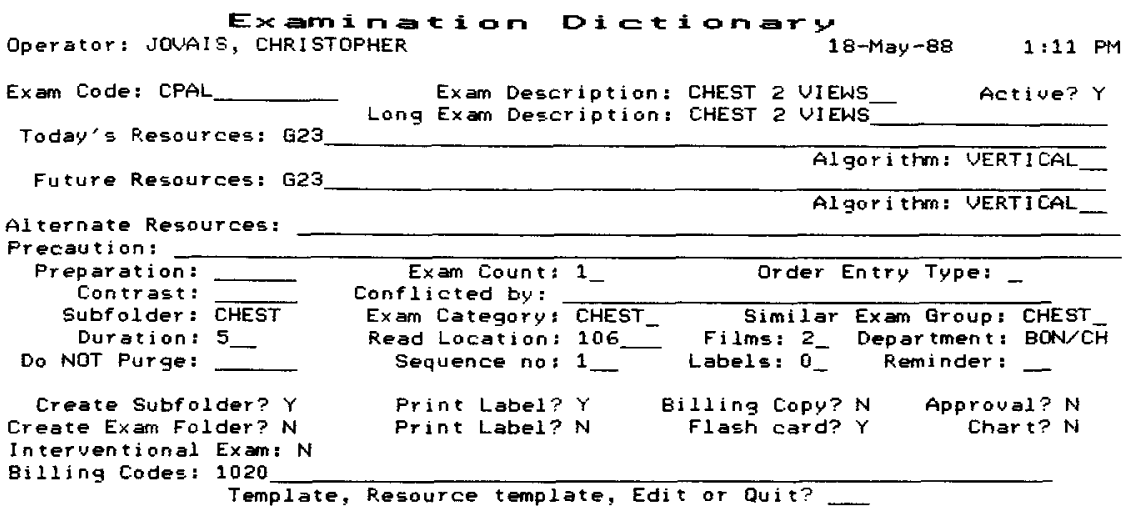

comparison prior to the patient's arrival. For exams scheduled in the future, the exam documents and pull notices are printed the evening before the scheduled date. The schedule lists are then distributed to the inpatient floors and the radiology staff uses the printed schedules which are organized by examination room.

Practically all patients are scheduled by telephone. Since clinical history or the reason for the examination is recorded at the time of scheduling, we are assured of some clinical information when interpreting the procedure. If a physician's secretary calls and does not have clinical information available, we politely tell them to call back when they have such data. Remember that the patient is still in the referring doctor's office and not in the radiology department. Although there were some concerns and complaints when this policy was introduced, there is very little difficulty at the present time.

If the patient has not been previously registered either through the hospital's registration and admission systems or in radiology directly, then the registration data is also obtained at the time of scheduling. This scheduling process starts the patient tracking functions. Once an exam has been scheduled, that exam must be completed and billed or it must be cancelled. If cancelled, the reason for the cancellation and the person cancelling the exam are recorded. If the exam remains in the scheduled status, it will appear on the "Scheduled But Not Yet Completed List" which is one of the exception reports in the system. Incidentally, the scheduler's initials are recorded with each procedure for accountability purposes. This logging is automatic since the scheduler's identity is known through the sign-on frame.

This scheduling system may seem complicated and unnecessary. Before and after implementing our scheduling system 11 years ago, we performed time-flow analyses to measure the effects of introducing such a system. ${ }^{5,6}$ The time interval between scheduling of procedures, such as barium enemas and urograms, and patient arrival was reduced from 55.3 hours to 27.2 hours after the introduction of the system. These findings paralleled those results measured previously at

ReEource schedule

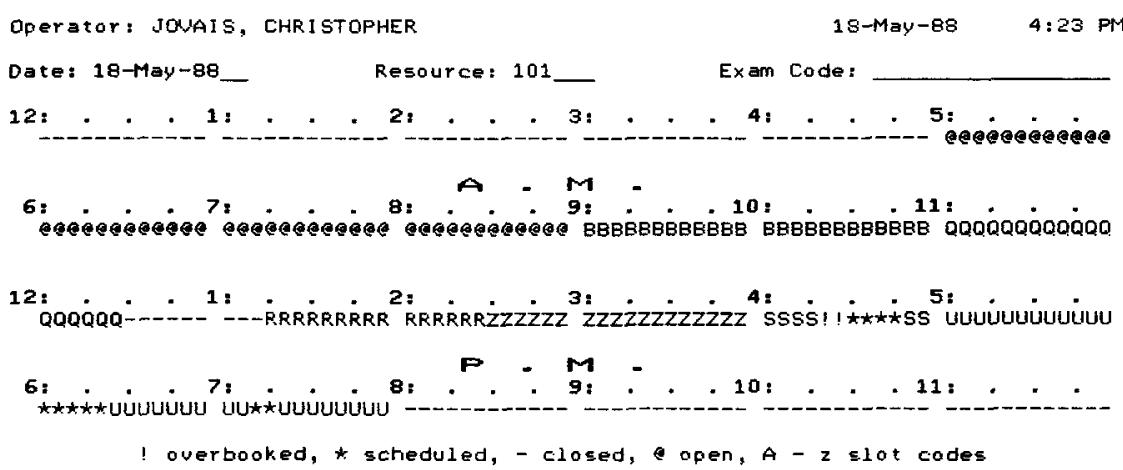

Operator: JONAIS, CHRISTOPHER
Fig 2. Room matrix with codes. This display shows the status of the time slots for one room, for one day. Each code entered into the five-minute time slots can represent a group of patient categories. An " $O$ " could allow any type of outpatient but an " $R$ " reserves the slot for emergency service patients. 


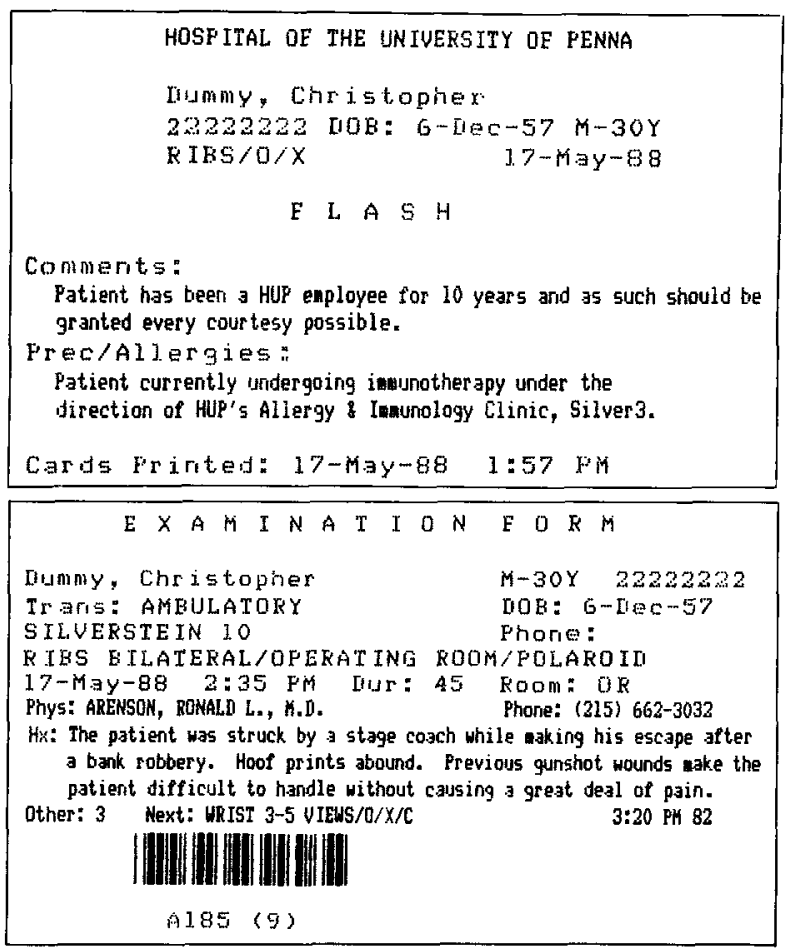

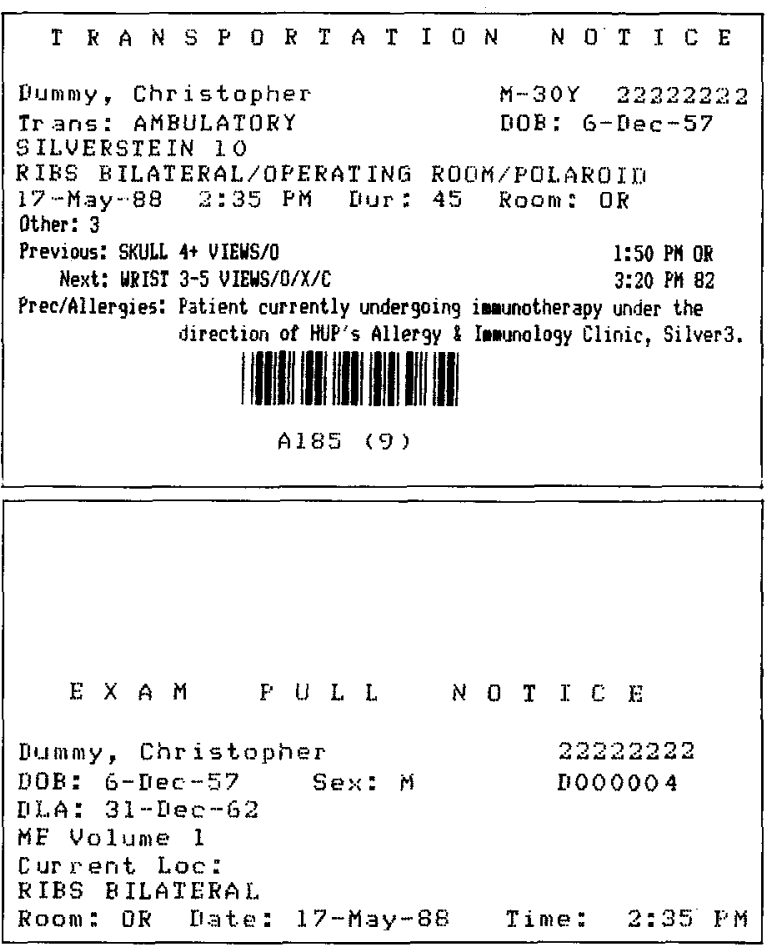

Fig 3. Exam documents, pull notice. (Top left) The flash card marks the patient data on the film itself. (Top right) The transportation notice is used by escort to pick-up and return the patient. (Bottom left) The exam card is used by the technologist to record, via bar-code pen, the exam completion. The radiologist uses the exam card for normal or "near normal" reporting. (Bottom right) The pull notice provides the film library with advance warning to "pull" previous films for comparison.

MGH. In addition, the interval of time between patient arrival and the initiation of the examination itself went from one hour to 20 minutes for inpatients and from 50 minutes to 35 minutes for outpatients. The interval from the beginning of the examination until the completion of the exam was reduced from one hour to 32 minutes.

The reductions in the scheduling delays were expected and are intuitively obvious. The marked reduction in the time that the patients spent in the department was not expected and is not so easily explained. Figure 4 shows the flow of patients into our main reception area prior to implementation of the computer system. The day is broken down into the number of patients who enter each hour, the averages, and the minimum and maximum numbers per hour. Obviously, there are wide ranges for each hour, with some maximums nearly three times the minimum numbers. Our assumption at the time was that we could handle approximately 30 patients per hour, which means that we exceeded our capacity for a portion of nearly every morning and every afternoon. The uncontrolled arrival of out- patients (mostly unannounced) would practically guarantee that we would be backed up.

What we did not know at the time was the significance of modern queuing theories. ${ }^{7}$ Table 1 shows the effects of uncontrolled patient arrivals in a simulated one-room facility. In the first column, an average of two patients will arrive every hour with the examination time expected to be 20 minutes, a capacity of three patients per hour. If one assumes a Poisson distribution for

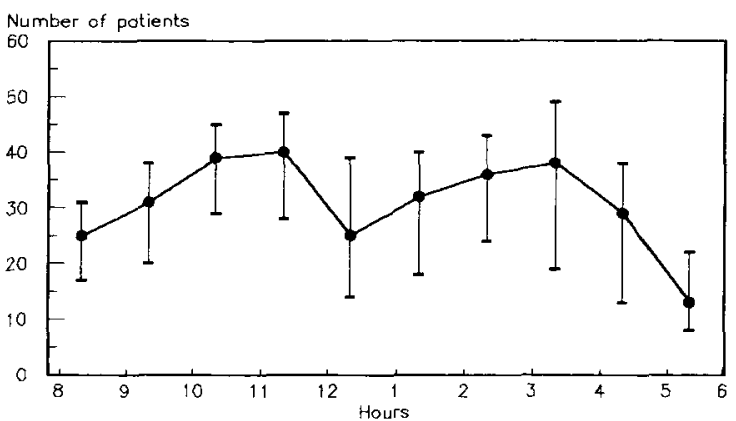

Fig 4. Patient influx before automated scheduling. The minimum, average, and maximum numbers of patients arriving in the main reception area are shown for each hour of the day. 
Table 1. Simulated One-Room X-Ray Facility

\begin{tabular}{lcc}
\hline & \multicolumn{2}{c}{ Patients Arriving per Hour } \\
\cline { 2 - 3 } & 2.00 & 2.44 \\
\hline Average time for procedure & $20 \mathrm{~min}$ & $20 \mathrm{~min}$ \\
Potential procedures per hour & 3.0 & 3.0 \\
Mean utilization (\%) & 66.6 & 81.3 \\
Expected number waiting & 1.33 & 3.5 \\
Expected waiting time per patient & $40 \mathrm{~min}$ & $86 \mathrm{~min}$ \\
\hline
\end{tabular}

patient arrivals and an exponential curve for the actual service time (average of 20 minutes), then at any moment in time one can expect 1.33 people in a queue (waiting) and an average waiting time of 40 minutes. The second column shows what happens if there is a modest increase in workload resulting in 2.44 patients arriving on the average per hour. Then the expected number in the queue will be 3.5 and the average time for waiting jumps to 86 minutes.

If the flow of patients into the department can be controlled by advance scheduling, then much greater efficiency can be expected from the facility, thus reducing the effects highlighted by the queuing theory. By reducing the average exam time from one hour to 32 minutes, we in essence doubled the size of our department. Scheduling in small departments is obviously much simpler than in very large ones. However, even a two- or three-room facility would benefit because of the queuing theory effects described.

Looking at our dedicated outpatient area, Silverstein 1 , we performed 40,814 exams last year and will perform 44,210 exams this year. Patient waiting has been kept to a minimum because of the scheduling system. The average interval from patient arrival until exam completion was 32 minutes last year, but rose to 39 this year.
Although this may not seem like a large change, it is rather significant. With an average of 14 minutes per exam (room time) in this dedicated outpatient area with six rooms, we are averaging 42.7 minutes per hour per room, which translates into $71.2 \%$ utilization. Any further increases in workload or any further shifts to peak hours will seriously undermine our ability to achieve efficient patient throughput. Our goal has always been to provide patient turnaround in 45 minutes or less. Therefore, we are increasing the facility by two rooms to provide adequate growth potential. Clearly many additional rooms would be needed without the scheduling system.

Another benefit of this degree of automation is the reduction in human error in both patient identification and exam scheduling. Training for schedulers is intensive, but they are not required to remember complicated rules for scheduling.

The reduction in unnecessary radiation exposure by avoiding duplicate exams turned out to be less than expected. A previous analysis at MGH suggested a 7\% reduction, but an analysis at HUP showed only a $2 \%$ decrease. This difference was probably due to the design of our inpatient request form, which lists previous exams scheduled in the patient's order book. In other words, physicians are becoming aware of duplication even before calling to schedule the exam.

In summary, the complete scheduling system described provides significant improvements in patient waiting, patient throughput, data accuracy, and clinical history availability, while improving quality of service through conflict checking. The advantages of the more sophisticated approach to scheduling is beneficial to both large and small departments.

\section{REFERENCES}

1. Lazarus CB, Poitras JW, Mitchell WP, et al: Automation of Scheduling and File Room Functions of a Diagnostic Radiology Department, vol I and II. Washington, DC, Department of Health, Education, and Welfare. Publication no. FDA 75-8020, February 1975

2. Bauman RA, Arenson RL, Barnett GO: Fully Automated Scheduling of Radiology Appointments: Proceedings of the Fourth Conference, Computer Applications in Radiology. Chicago, American College of Radiology, 1975, pp 461-468

3. Arenson RL, London, JW: Impact of Automated Radiology Scheduling: Proceedings of Second Symposium in Computers in Diagnostic Radiology/Third International Symposium on the Planning of Radiological Departments.
The Hague, Netherlands, SMITS, Drukkers-Uitgevers, 1980, pp 490-492

4. Bauman RA: The registration and scheduling process. Radio Clin North Am 24:5-9, 1986

5. Arenson RL, London JW: Comprehensive analysis of a radiology operations management computer system. Radiology 133:355-362, 1979

6. Arenson RL: Automation of the radiology management function-Symposium on digital computers in high tech radiology. Radiology 153:65-68, 1984

7. Rosenquist CJ: Queuing Analysis for Planning and Management in Radiology: Proceedings of the Ninth Conference, Computer Applications in Radiology, Radiology Information System Consortium (RISC). 